\title{
Computational Design and Construction of Notch-free Reciprocal Frame Structures
}

\author{
Nicolas Mellado \\ University College London \\ Peng Song \\ University of Science and Technology of China \\ Xiaoqi Yan \\ Nanyang Technological University \\ Chi-Wing Fu \\ Nanyang Technological University \\ Niloy J. Mitra \\ University College London
}

\begin{abstract}
.
A reciprocal frame $(R F)$ is a self-standing $3 D$ structure typically formed by a complex grillage created as an assembly of simple atomic $R F$-units, which are in turn made up of three or more sloping rods forming individual units. While RF-structures are attractive given their simplicity, beauty, and ease of deployment; creating such structures, however, is difficult and cumbersome. In this work, we present an interactive computational framework for designing and assembling RF-structures around a $3 D$ reference surface. Targeting notch-free assemblies, wherein individual rods or sticks are simply tied together, we focus on simplifying both the process of exploring the space of aesthetic designs and also the actual assembly process. By providing computational support to simplify the design and assembly process, our tool enables novice users to interactivity explore a range of design variations, and assists them to construct the final RF-structure design. We use the proposed framework to design a range of $R F$-structures of varying complexity and also physically construct a selection of the models.
\end{abstract}

Project page: http://geometry.cs.ucl.ac.uk/projects/2014/rf-aag 

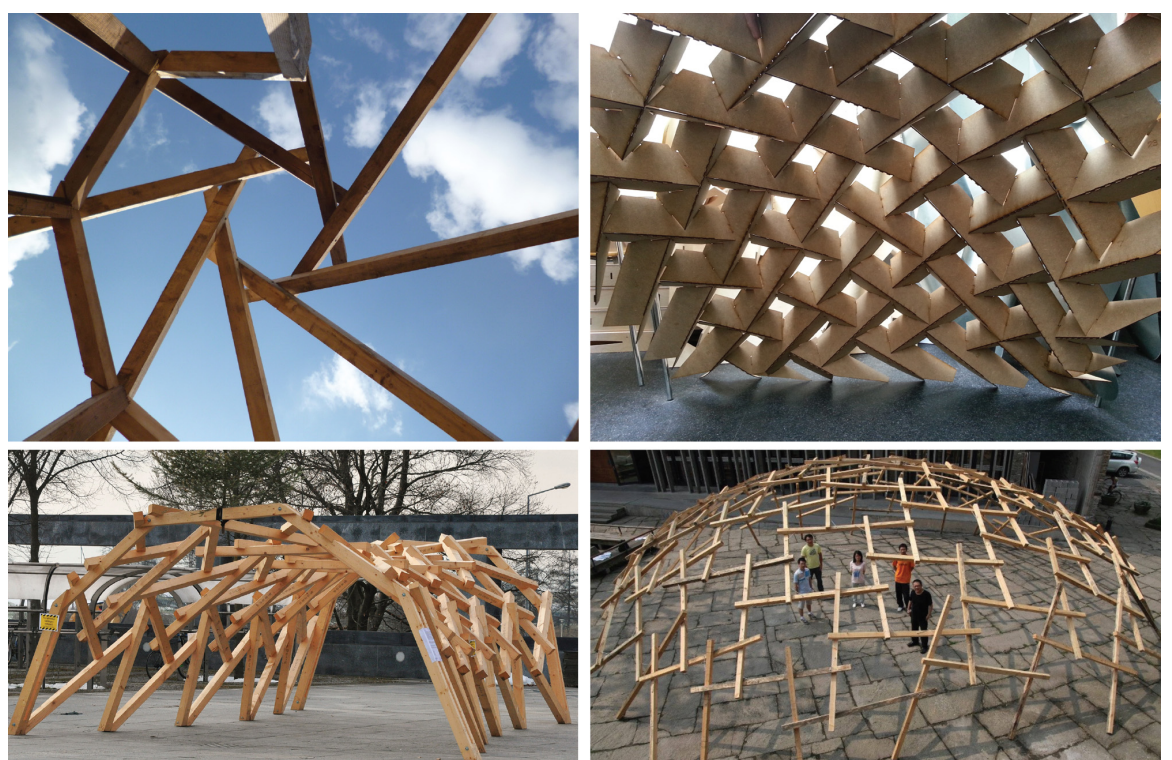

Figure 1: Physical RF-structures designed and fabricated by architects: a simple roof structure (top-left), a design by Michael Clarke (top-right), a design from Spiro-ETH (bottom-left), and a design from Wan Shu and Kengo Kuma (bottom-right).

\section{Introduction}

Reciprocal frame (RF) is a 3D assembly of mutually-supported rods without any central support pillars. Such an assembly structure usually consists of simple atomic units called RF-units, which are three or more slanted rods supporting one another in closed circuits. The inner end of each rod rests on and is supported by its adjacent rod, and a closed circuit is obtained as the last rod is placed over the first one in a mutually-supporting manner. At the boundary, such structures are supported by pillars, walls, or ring beams.

Beyond structural considerations, RF assemblies are simply beautiful and elegant. For centuries they have been used as roof or floor patterns in churches, or in construction of traditional houses in Japan and China (c.f., [Chilton 1995; Larsen 2008]). Such designs, however, were largely restricted to flat grillage patterns (e.g., sketched by Honnecourt, Leonardo da Vinci) or circular plans, i.e., the rods meeting at one point in the center (e.g., an Indian tent). In the recent decades, RF-structures have inspired many architects and structural engineers, rekindling interest in age-old principles in structural systems. Moreover, emerging applications of computational optimization and CAD tools have inspired researchers to enhance, enrich, and scale up RF-structure construction [Pugnale et al. 2011]. Figure 1 shows a few examples.

In this work we focus on notch-free RF-structures, which involve rods (e.g., bam- 
boo sticks) that are simply tied together with strings, as opposed to wooden or metal beams joined at complementary grooves. The notch-free structures being light-weight, the binding threads are sufficient to balance the frictional forces to keep the rods in place. Further, being made of simple rods tied together, these RF-structures are highly cost-effective deployable systems, particularly suitable for rapid constructions of temporary structures (see [Larsen 2008] for more details).

Creating such structures consists of two main stages: a design phase to specify the RF-structure spanning a given reference surface, and a construction phase to actually realize the designed structure tying together the sticks in an appropriate assembly sequence. The design phase comprises of selecting and laying out RF-units, and deciding their inter-connections. In classical tent-like roof design, the process is quite simple: a single RF-unit with only design freedom being the number of rods spanning the inner circle (see Figure 1 top-left). The key technical challenge is to design for more general reference surfaces, while adopting a wide variety of RF-units. Although the design space is large, manually exploring such a space is quite cumbersome, and demands expertise in both aesthetics and engineering. Classical examples are the various architectural designs by Leonardo Da Vinci during the Renaissance period.

Furthermore, the construction phase imposes certain assembly constraints: (i) the rods or sticks should be straight and appropriately overlap at the junctions (i.e., avoid intersections or gaps); and (ii) the structure should be stable and should not fall over in the absence of suitable supports. More importantly, during the assembly process, the partial structure should also remain stable under self-weight, or appropriate intermediate support pillars, which are referred to as Charlie sticks, should be added to restore stability. Such constraints are difficult to manually account for in case of medium- or large-span RF-structures, as in the focus of this work.

We provide an interactive tool to aid the design phase of RF-structures. Based on the observation that RF-structures are self-similar and form highly symmetric patterns spanning vast architectural spaces, we establish a duality between RF-structure patterns and planar tiling theory, which is mathematically well understood and characterized. Based on the recent work by [Song* et al. 2013], we split the design phase as: a planar grillage design using RF-units arranged according to various tiling patterns; and a conformal lifting phase to morph the planar grillage to the reference surface subject to various fabrication constraints.

In order to simplify the assembly process, we propose multiple strategies to determine an effective assembly sequence for the designed RF-structures, while also proposing respective insertion and removal sequence of supporting pillars. The strategies are formulated by computationally evaluating the feasibility of the intermediate RF-structures based on physical stability tests and scoring the validity of the intermediate structures. Specifically, the system proposes both the sequence of rods to be added and the placement of intermediate support pillars (see the supplementary video and Figure 10). This vastly simplifies the assembly process since our 


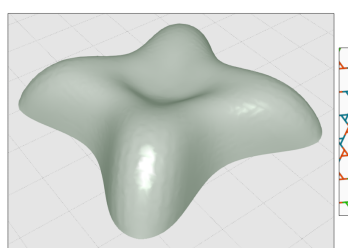

(a)

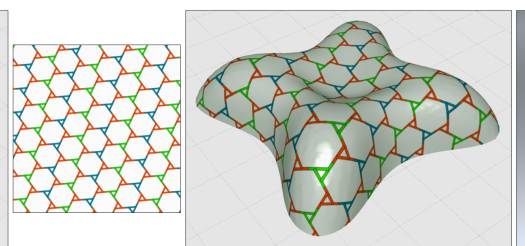

(b)

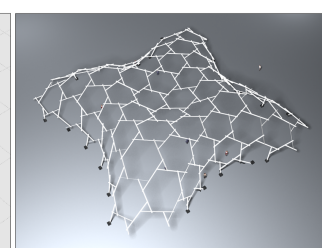

(d)

Figure 2: Overview of the design phase: (a) reference surface; (b) planar grillage (RF tessellation pattern); (c) conformal mapping; and (d) final optimization (non-intersecting).

system automatically proposes different assembly sequences, while accounting for different fabrication constraints.

Using our interactive framework, users can quickly sketch and formulate designs with extended number of RF-units, allowing them to easily manipulate, test, and preview a wide variety of coherent RF-patterns with feasible geometric parameters. We used our tool for designing various RF-structures by varying the grillage patterns and the reference surfaces. We physically constructed a selection of designs by assembling them using the guidance provided by our computational tool.

\subsection{Related Work}

Background. For centuries reciprocal frames have been used in design and construction, e.g., the classical bridge sketches by Leonardo da Vinci, the roof of Nagasaki Castle in Japan, as well as Eskimo tents. However, we generally lack computational support to design and construct RF-unit-based structures. Hence, most existing realizations are restricted to small structures involving only a few RF-units. Current practice cannot be generalized to handle larger structures due to various challenges involving where to place the RF-units, how to interconnect them, and how to realize a meaningful aesthetic design.

RF-structures in practice. Architects often manually experiment with different ways of assembling RF-units by testing physical mock-ups created using rods (e.g., in a scale of 1:5 [Chilton 2009; Gelez and Saby 2011]. Although such an approach gives full control over the design, form finding remains challenging while ensuring a valid arrangement of the RF-units. As a result even relatively simple designs can be tedious and very time-consuming to mock-up.

[Pugnale et al. 2011] stressed the need for computational tools for RF-based designs. Existing attention, however, is focused on handling the engineering issues such as force analysis on the structural stability [Douthe and Baverel 2009; Kohlhammer and Kotnik 2010] and the fitting of rods to form a connected RF [Baverel et al. 2004; Parigi et al. 2012]. [Brocato and Mondardini 2010] proposed a geometric method to design stone domes with extended number of RF-units, but their method 
supports only one class of RF-patterns and offers a few parameters for user control. [Thönnissen and Werenfels 2011] employed a Rhino-script to aid students to design RF-structures and arranged the RF-units over the cells obtained as the Delaunay triangulation of points on the input reference surface. However, since the point set can have arbitrary distribution, the resulting RF-structures can be rather irregular. Further, the users have little control on the RF-patterns, and have no support to interactively preview and refine their designs.

Fabrication-aware form finding. In a more general context of architectural geometry, different geometric modeling methods have been proposed for different fabrication constraints. [Whiting et al. 2009] explored structural feasibility in the context of modeling masonry buildings. They proposed a gradient-based optimization method to search the parameter space in procedural models to generate stable buildings, and recently [Whiting et al. 2012] introduced a stability metric and optimized building geometry to achieve stability. Concurrent efforts [Fu et al. 2010; Eigensatz et al. 2010; Singh and Schaefer 2010] introduced cost-driven methods to analyze and optimize panel types and shapes towards cost-effective constructions of free-form surfaces. [Yang et al. 2011] devised a computational framework to characterize, parameterize, and navigate non-linearly constrained shape spaces to access feasible designs that satisfy a given set of constraints.

Computational design tools. With growing focus on physically-manufacturable objects, e.g., papercraft models [Mitani and Suzuki 2004; Li et al. 2010], or interlocking 3D puzzles [Xin et al. 2011], there has been increased effort in developing computational design tools. [Umetani et al. 2012] proposed a design suggestion interface that employs a force-analysis model to guide users to design valid shapes of furniture models under geometric and physical constraints.

Construction sequences. Despite the growing interest in architecture geometry, the optimization of construction sequences is today mostly unexplored. As far as we know, only two recent works study this topic, in the context of planar-pieces assemblies [Schwartzburg and Pauly 2013; Cignoni et al. 2014]. These approaches are, however, tailored for slit assemblies, and cannot be directly applied to RFstructures.

In this work, we develop a computational tool to support design and assembly of large RF-structures, which are difficult to conceive using physical mockup-based experimentation. In our tool, one can quickly sketch up an RF-structure by combining different RF-units, flexibly modify its appearance and pattern, as well as interactively experiment with different design parameters while the underlying optimization ensures connectivity and structural coherence. Assembly sequences are then interactively explored by combining criteria specific to RF-structures (e.g., rods are added by units), self-supporting structures (e.g., balance is ensured at each step) or any other specific construction criteria (e.g., aesthetic). 

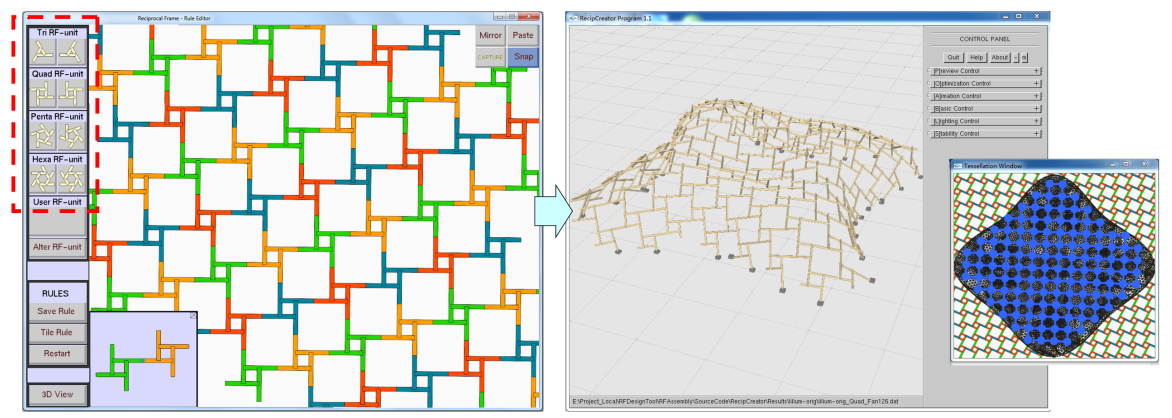

Figure 3: User interface of the proposed RF-structure design tool. Left: the RF Pattern Editor for designing 2D RF-tessellation. Right: the RF Creator for designing the 3D RFstructure; the inset reveals the conformal map.

\section{Overview}

Our interactive computational tool considers two aspects: design and assembly. Figure 3 shows a preview of the design/assembly tool.

For the design aspect, our tool allows the user to create RF-patterns with selfsimilarities by tiling with basic construction units. A basic set of building blocks (see Figure 3 left), which we call RF-units, are offered to the user, but the user can further design and customize them if she likes. The units are used to tessellate a plane (see Figure 2(b)), before (conformally) lifting them to 3D around a prescribed reference surface (see Figure 2(c,d) and Figure 3 right). In order to guarantee that the RF-tessellation is coherent, the system first validates that a 2D tessellation can be generated from the RF-units specified by the user; then, it optimizes the 3D RF-structure to further ensure that the resulting grillage is coherently-connected in $3 \mathrm{D}$, that is, the original angles between the rods of the RF-units are preserved, and the rods are properly touch in 3D space without self-intersections or gaps. Thus, our system by providing interactive feedback, readily allows the user to design and visualize large RF-structures for a given guiding surface.

The generated RF-structure is a parametric model. The user can interactively modify its parameters, preview its appearance on a guiding surface, and easily experiment with different design variations. Further, the tool assists in the assembly planning phase. Beyond enumerating the stick lengths, the tool also provides guidance pertaining to the sequence of assembling the rods and also prescribes additional (intermediate) support pillars, if necessary. The user can select among different strategies ranging from greedy ones (suitable for simple designs) to more advanced ones optimized for functional validity (i.e., basic stability test in this work). 


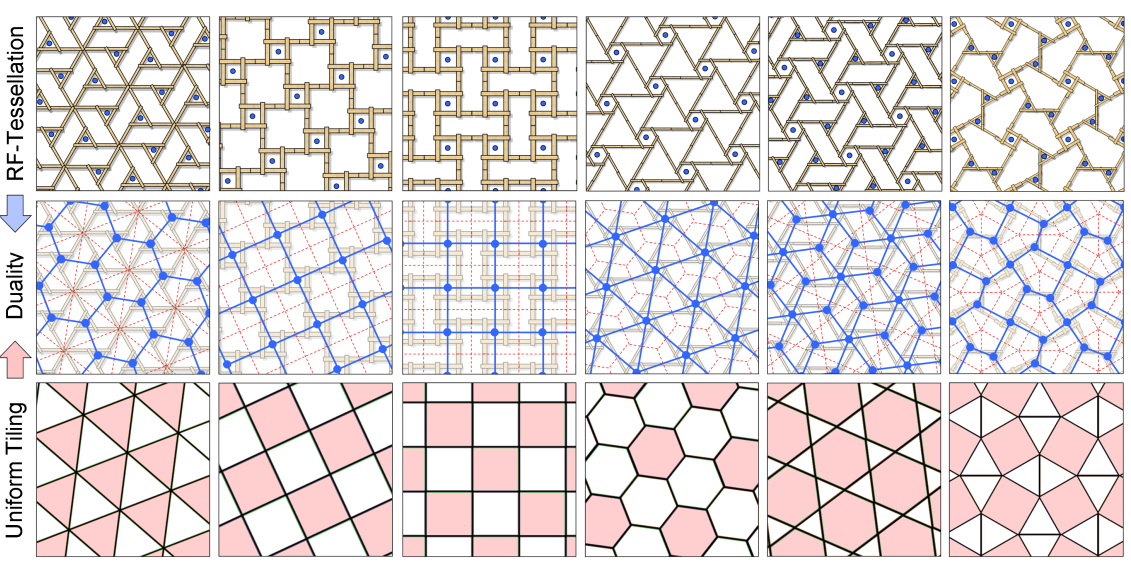

Figure 4: Duality: RF-tessellation pattern (top) is a dual of uniform tiling (bottom).

\section{RF-structure Design}

In this section, we briefly summarize three key components of the RF-structure design phase: from the design of RF-patterns to the optimization of rods arrangement in 3D (see also [Song* et al. 2013]).

\subsection{Duality between RF-Tessellation and Plane Tiling}

Our first contribution is on connecting RF-structures with plane tiling [Grünbaum and Shephard 1986]. We found that an RF-tessellation with rotationally-symmetric $R F$-units is structurally-equivalent (dual) to an edge-to-edge tiling by congruent regular polygons. From Figure 4, we can see that if we replace faces and edges in a uniform tiling by RF-units (individual fans of reciprocal frames) and their connections, respectively, we can obtain the large RF-structures shown on top of the figure. By drawing such an analogy, we can achieve the followings:

- first, we can define grammar rules (see Figure 5) to procedurally and effortlessly generate RF-structures according to the related tiling pattern;

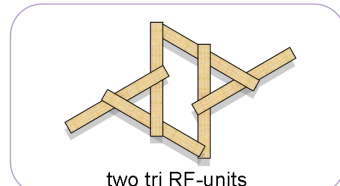

two tri RF-units

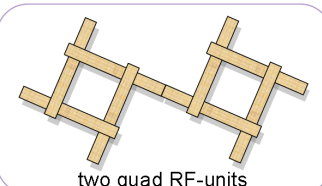

two quad RF-units

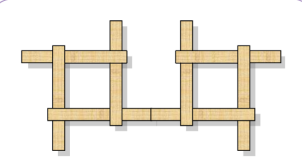

two quad RF-units

Figure 5: The grammar rules that define the three left-most RF-structures in Figure 4. 
- second, we can efficiently testify the validity of a given grammar rule, and predict whether the rule can generate coherent RF-tessellations; and

- lastly, we can manipulate a grammar rule and re-position RF-units in a complex RF-structure, thus enabling interactive exploration of RF designs (see Figure 6).

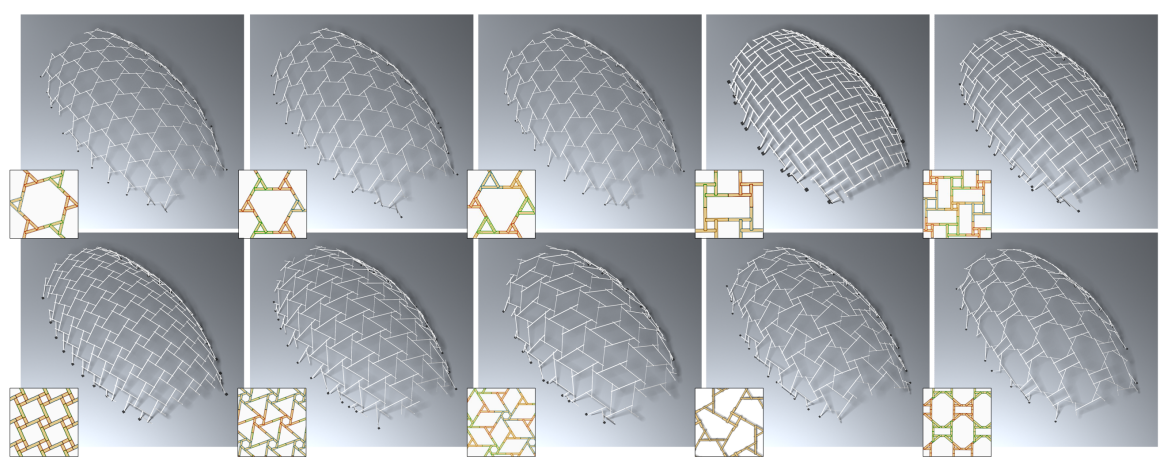

Figure 6: Different designs realized on the CANOPY reference surface using different choice of RF-units, shown as insets.

\subsection{Lift RF-Tessellation to 3D}

Our second contribution is a fast method that lifts a 2D RF-tessellation over a 3D reference surface (see Figure 2(a-c)). Here we first compute a conformal map of the given reference surface by the ABF++ method [Sheffer et al. 2005], so that we can maintain the structural symmetry during the lifting operation. Then, we construct an approximate RF-structure in 3D by inversing the conformal map and lifting up each rod from the 2D RF-tessellation into the 3D space (see Figure 3 right). Though this method may not produce coherent RF-structures with proper rods contacts, it enables high-level RF designs, where we can interactively explore different RF parameters while seeing the changes in the approximate RF-structure.

\subsection{Optimize the Rods Arrangement in 3D}

Since the lifted rods may penetrate or slightly float above one another in the approximate RF-structure, we use an optimization to solve for intersection-free rods placements in 3D, while adhering to the initial design. This is a two-level optimization scheme. First, we relax the RF-units centers on the reference surface to secure a better initial rods arrangement in 3D. Then, we iteratively optimize the rods positions in 3D by formulating an objective function with the rods contacts constraint 
$\left(F_{1}\right)$, surface constraint $\left(F_{2}\right)$, and conformality constraint $\left(F_{3}\right.$ and $\left.F_{4}\right)$ :

$$
\begin{array}{cl}
\min _{\text {all rods }} & \alpha \sum_{i, j} F_{1}^{2}\left(P_{j}^{i}, P_{i}^{j}\right)+\beta \sum_{\left\{V_{i}\right\}} F_{2}^{2}\left(V_{1} V_{2} \cdots V_{m}\right) \\
\quad+ & \gamma \sum_{i} F_{3}^{2}\left(V_{i-1}, V_{i}, V_{i+1}\right) \zeta \sum_{i} F_{4}^{2}\left(V_{i}, V_{i+1}\right)+\lambda \sum_{i}\left(P_{i}-\bar{P}_{i}\right)^{2},
\end{array}
$$

and then linearizing and solving this minimization problem by a least-squares method. Please refer to [Song* et al. 2013] for symbol notations and details.

\section{RF-structure Assembly Sequence}

\subsection{Problem Formulation}

Like any other structure, the assembly and construction of an RF-structure is constrained by physical laws, inducing both global and local constraints, e.g., respectively structure balance or material tensile strength. Simulations can be ran on entire structures, however they do not take into account the temporary constraints that could be applied during the construction stage. In contrary to self masonry structures that may need a supporting shape to be constructed [Panozzo et al. 2013], RFstructures are made of multiple self-supported components, that can be assembled in various order to produce the final RF-assembly. Depending on the order chosen to assemble the RF-units, the building can suffer during construction from various issues, e.g. unbalanced assembly or local constraints beyond material resistance.

In this section we propose a generic formulation to produce construction sequences and avoid invalid intermediate assemblies. Our approach takes as input a valid RFstructure, and output a list of constructions steps, including intermediate supports to stabilize the structure when it is unstable.

Our formulation is elaborated around construction strategies, which define how to split an input structure as construction elements, and in which order they should be assembled (see Section 4.2). We model the physical constraints that must be respected during the construction as cost functions. They are used by strategies to disambiguate between different solutions and ensure the feasibility of the construction sequence, eventually by suggesting intermediate supporting elements like pillars (see Section 4.3).

\subsection{Construction Strategies}

The aim of a construction strategy is to decide how to split an RF-structure into construction elements, and explore the different assembling alternatives with respect to a given cost function. We define in this paper two ways to split and assemble RFunits: gradually or by group, leading the names GRAD and GROUP (see Figure 7). With GRAD strategies, fans are added one by one, for instance, by following a counter-clockwise order in each successive $n$-ring surrounding the starting point. 

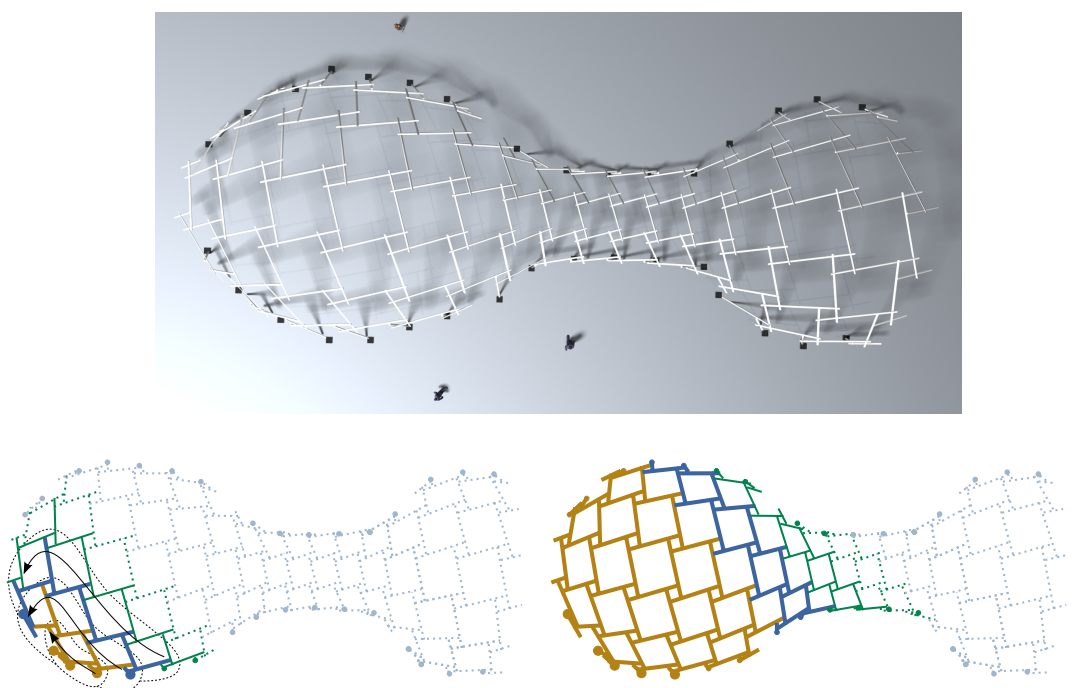

Construction steps

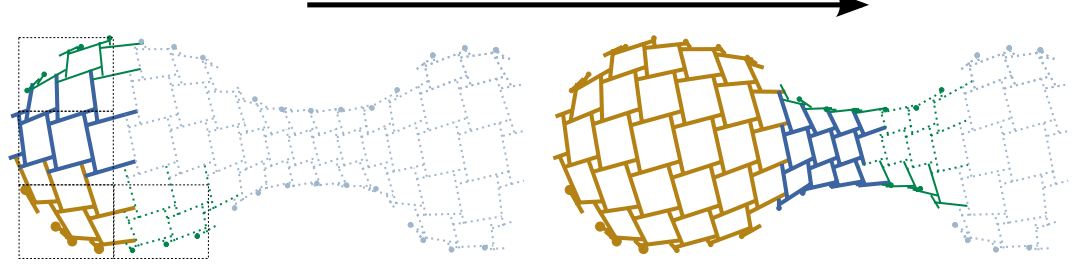

Figure 7: (Top) Top view of the PEANUT model; (bottom) two different construction strategies, where RF-units are added from a starting point either gradually (GRAD strategy, following $n$-rings and counter-clockwise order), or by groups (GROUP strategy)

. The model on the top uses orthographic projection, and successive steps are visualized in gold, blue and green.

Instructions to build a structure are elaborated as follow: a user select a starting RFunit to erect, a strategy that must be used (GRAD or GROUP), and a cost function. Starting from the user selection, other RF-units are iteratively marked as constructed and associated instructions generated. Invalid configurations are also detected at this step to avoided when possible, or fixed using pillars.

A crucial question is here to define in which order to pick unselected RF-units surrounding those already erected, and construct them. We propose two solutions, that can be applied with both GRAD and GROUP approaches. A first solution is simply to add RF-units using a predefined regular order, either along the selected region for GRADREG or using coordinates in the slicing grid for GROUPREG (see Figure 8 (a-c)). Here, the cost function is used only to check the configuration validity and 


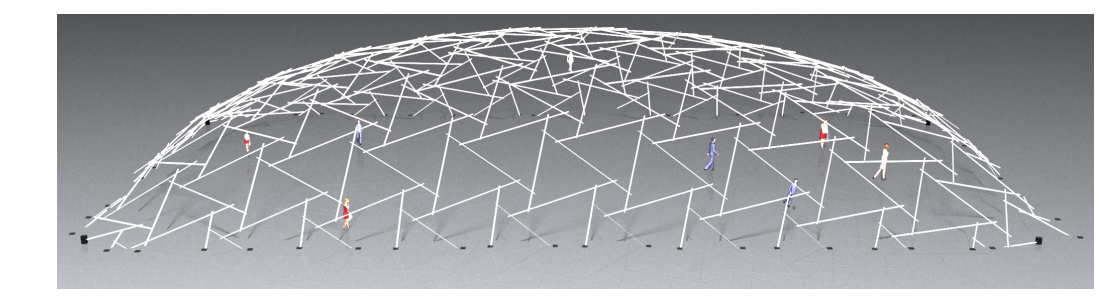

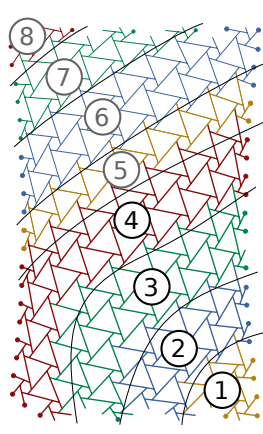

a)

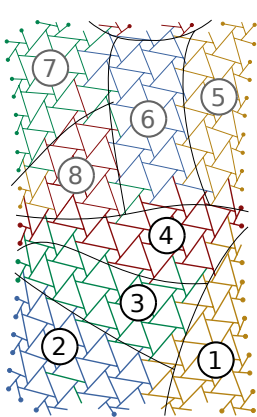

b)

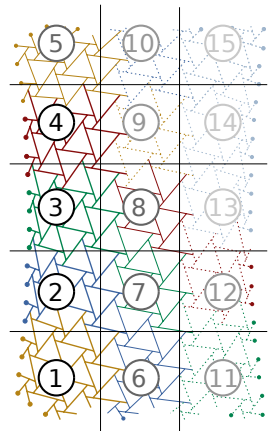

c)

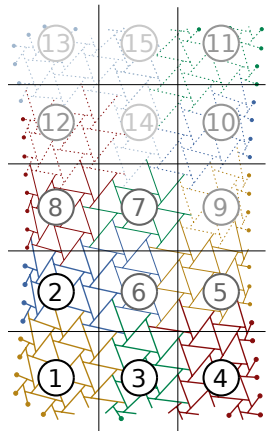

d)

Figure 8: Proposed building strategies. Approaches (a) and (c) use the cost function for verification and to suggest support pillars; while greedy approaches (b) and (d) use the cost function to also determine the assembly order.

suggest pillars.

A second solution is to create a candidate configuration for each of the RF-units that could be added, analyze it using the cost function, and greedily select the configuration introducing the smaller cost. These approaches GRADGREEDY and GROUPGREEDY are illustrated in Figure 8 (b-d). Potentially invalid configurations can still be added only when fixed using temporary pillars suggested by the cost function.

\subsection{Cost Functions and Supporting Elements}

As described in the previous section, designing cost functions is a critical step in order to obtain suitable construction sequences: they are used to detect invalid configurations, chose between valid ones, and suggest temporary pillars.

In our system, a cost function takes as input an RF-structure and offers to: a) compute its cost and detecting invalid configurations, b) suggest pillars positions. Thanks to this generic formulation, we can plug any analysis in our system, ranging from simple balance check to accurate physical simulations. However, we demonstrate the validity of our approach with two simple cost functions, sufficient in our case to construct real models (see Section 5).

Our first cost function aims at favoring RF-units close to the ground, by returning 

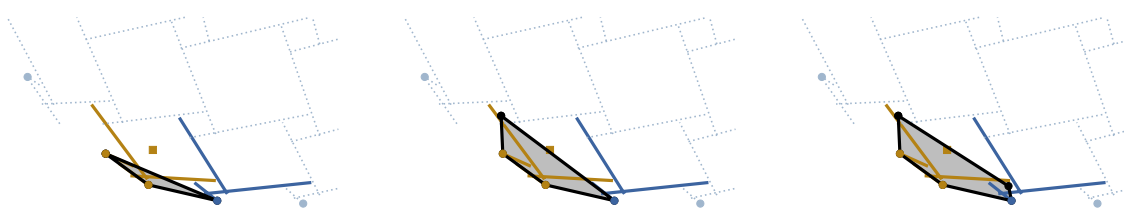

Figure 9: Support pillar suggestion to stabilize an RF-structure (shown from the top using orthographic projection) at the beginning of its construction: golden and blue units form an unbalanced RF-structure, where their gravity center (golden square) is out of the polygon (gray) formed by its support points. Two successive pillars insertion are necessary to restore stability. Next, RF-units are shown as dashed lines.

the height of potential candidates as a cost. Neither pillars nor invalid configurations are used here, so its use is limited to simple RF-structures with few elements.

Our second cost function analyzes the balance of an RF-structure to be sure it will not fall down at some point. To detect such situations, we extract the convex 2D polygon defined by the position of the supporting rods on the floor. Then, we compute the gravity center of all the rods of the structure, and check if it is vertically projected inside the convex polygon or not.

In the first case, the RF-structure is stable, and the cost is measured as the distance from the gravity center to the convex polygon, normalized by the area of the later. This normalization is critical to avoid favoring configurations with elongated configurations.

In the second case, illustrated in Figure 9, the RF-structure is unbalanced. Supporting pillars are then suggested below the farthest RF-unit, until a stable configuration is obtained.

Our system also supports combining cost functions. We used this functionality to generate LILIUM and INNER COURT BUBBLE in Figure 12 in order to add RF-units close to the floor first and avoid unbalanced configurations thanks to temporary pillars.

\section{Results}

Our tools are implemented in C++ and OpenGL. The main tool is dedicated to the design and assembly planning of RF-structures and it consists of two main panels (see Figure 3): (i) RF Pattern Editor, for composing and editing RF-units and grammar rules; and (ii) $R F$ Creator, for making RF designs with interactive editing and for performing the optimization. A second command line tool takes as input the files describing an RF-structure and computes the construction steps with needed (auxiliary) pillars, and producing the instruction list for fabrication. Both tools ran at interactive rates for all the tested models. 
Table 1: Statistics of RF-structures shown in Figure 12.

\begin{tabular}{|c|c|c|c|c|}
\hline Reference Surface & RF Pattern & \# fans & \# rods & \# pillars \\
\hline \hline HYPERBOLIC & Triangular Fans & 32 & 55 & 0 \\
\hline HYPERBOLIC & Quad Fans & 12 & 31 & 4 \\
\hline INNER COURT BUBBLE & Hex Fans & 84 & 288 & 2 \\
\hline LILIUM & Quad Fans & 126 & 280 & 1 \\
\hline PEANUT & Quad Fans & 80 & 184 & 2 \\
\hline
\end{tabular}

RF-design. Our tools support a wide variety of RF-patterns, and their variations. Our interactive design tool allows users to apply RF-patterns on a $3 \mathrm{D}$ reference shape and to test various parameter configurations (see supplementary video ${ }^{1}$ ). Figure 6 shows ten RF-structures generated with different RF-patterns on the CANOPY reference surface. Our tool can be used to design RF-structures over guiding surfaces of many different shapes. Figure 12 shows final results using HYPERBOLIC, InNer Court Bubble, Lilium, and Peanut, while Table 1 lists the associated statistics.

RF-construction strategies. Our second tool allows users to elaborate the construction instructions for any kind of RF-structure, independently of its RF-pattern and guiding shape, as illustrated in Figure 12 (see complete sequences in supplementary video). Regarding to its needs and constraints, one can choose to prioritize the RF-structure construction with respect to the RF-units height (HYPERBOLIC, triangular fans), or to run at each step a stability check, use this criterion to sort the units and suggest intermediate pillars (HYPERBOLIC, quad fans). Both criteria are combined to generate the construction sequences for LILIUM and INNER COURT

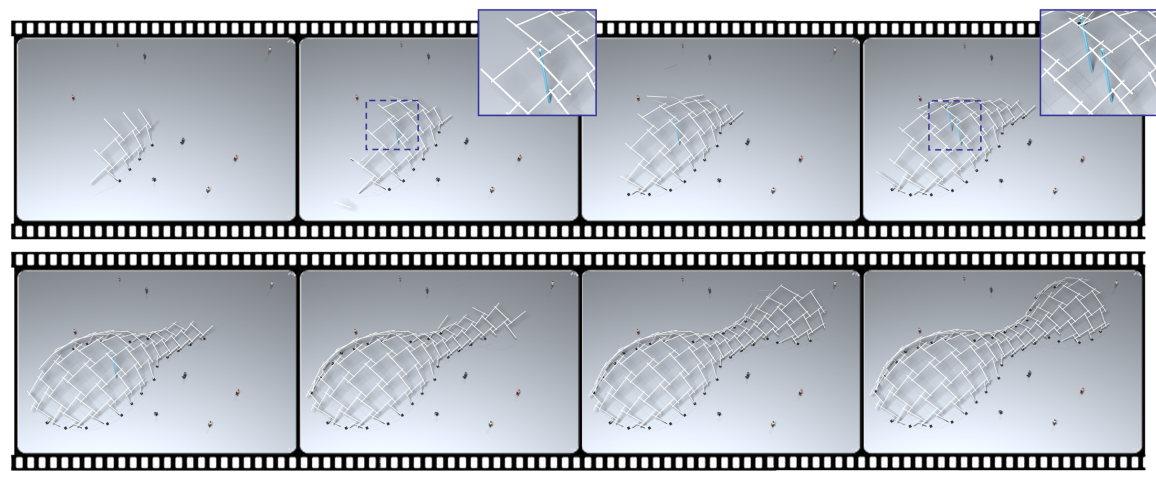

Figure 10: Snapshots of the assembly sequence for the PEANUT model. The proposed support pillars are in blue.

${ }^{1}$ The supplementary video can be accessed on the project page: http://geometry.cs.ucl.ac.uk/projects/2014/rf-aag/ 


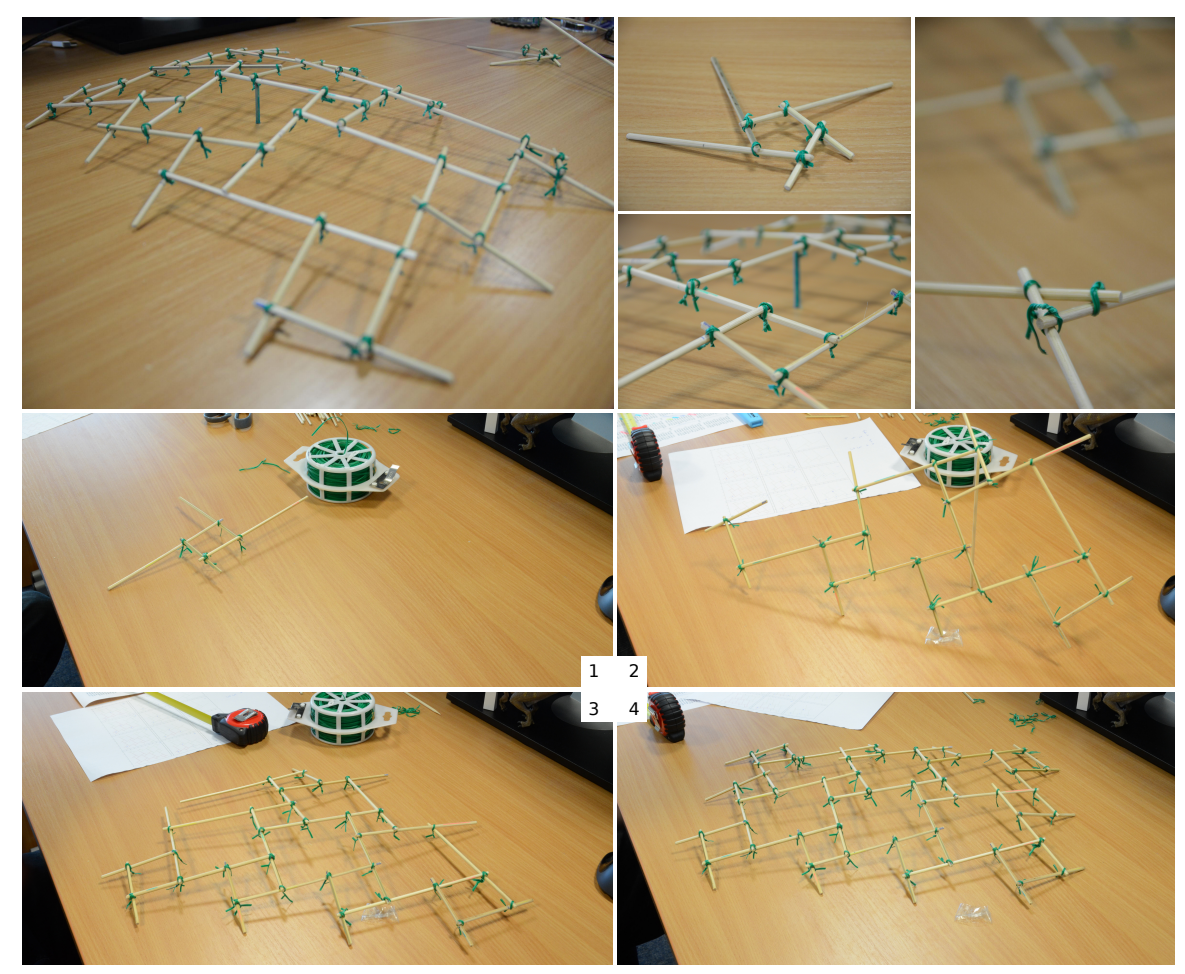

Figure 11: Physical constructions with bamboo sticks. Top-left: a complete structure, with intermediate steps (bottom). Top-right: close-up view of a triangle, quad, and penta RF-unit.

BUBBLE, permitting us to first assemble the supporting RF-units, add pillars when necessary, and then build the roof. Note that when used in isolation, the stability cost can produce unstable configurations. In such cases, the system proposes addition of temporary pillars to restore stability (see Figure 10). The temporary pillars can later be removed as later rods are added to restore stability as the later structures extend the building.

In addition to cost functions, our tools allows different strategies, such as regular slicing or growing strategies (see Figure 7). Impacts on the construction instructions are shown for PEANUT in Figure 8 and in the supplementary video.

By adapting the cost functions and construction strategies, ones can experiment with various and practical instruction sets to build RF-structures. In our experiments, computation work in real time, making strategy exploration feasible even for complex models comprising of hundreds of units. Figure 11 shows an example of physical construction and the intermediate steps. The physical assembly time was three hours for a completely novice user, including rod cutting. 


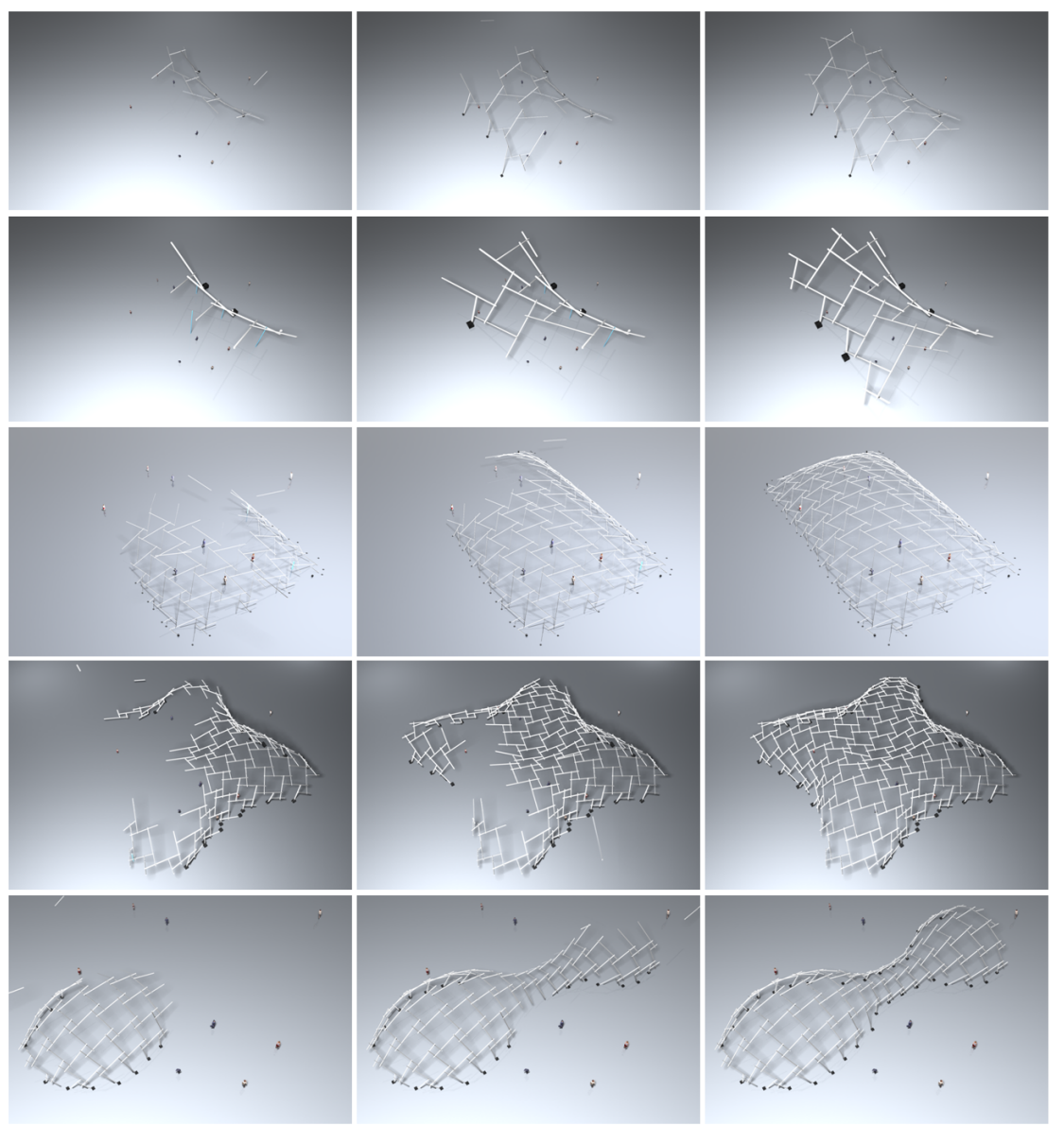

Figure 12: Assembly process of five different RF-structures, corresponding to the five data rows in Table 1 (from top to bottom).

We observed a few failure cases during our experiments, where our system fails to produce a construction sequence respecting the input constraints. Each time, it was due to unbalanced configurations that could not be stabilized even by adding pillars. Such situations arise rarely, always during the early assembly steps, where there are only a few RF-units, and we can usually correct it by selecting a new starting point. A more general solution would be to allow adding pillars below rods extremities instead of units centers only. We leave this as future work. 


\section{Conclusion}

We presented an interactive tool to facilitate the design and assembly of mid- to large-scale RF-structures. In the design phase, by establishing a connection between RF-structure layout and the classical tiling theory of the plane, we propose an interactive design framework to allow users to bring together simple RF-units to form intricate design patterns over 3D reference surfaces. In the background, the computational tool automatically enforces basic fabrication constraints arising from notch-free constructions. Subsequently, we proposed and investigated several assembly strategies to aid in the construction of such large-scale structures, assisting in determining both the assembly sequence and also the location and placement of intermediate support pillars. We demonstrated the usefulness of the computational tool on several case studies on reference surfaces of varying geometric complexity.

In this work, we do not take into consideration the effect of force and torque on the RF-structures, neither on the final structure, nor during the intermediate construction stages. Since, a common problem with RF-structures involves sagging leading to ultimate collapse of the structure, in the future, we plan to investigate this problem and develop computational tools to appropriately reinforce the designs. We would also like to algorithmically consider the effect of the dimensional of the working environment and take into consideration the ease of manipulating the RF rods that could be heavy and difficult to maneuver, especially in narrow and restricted spaces. Finally, we believe that our formulation is generic enough to be adapted to the construction of other structure types, like planar-pieces assemblies [Cignoni et al. 2014].

\section{Acknowledgements}

We thank the reviewers for their comments and suggestions for improving the paper. We thank Moos Hueting, James Hennessey and Aron Monszpart for their help on physical construction and discussions. This work was supported in part by the Marie Curie Career Integration Grant 303541, the ERC Starting Grant SmartGeometry (StG-2013-335373), the MOE Tier-2 grant (MOE2011-T2-2-041 (ARC 5/12)), and gifts from Adobe Research. 


\section{References}

Baverel, O., Nooshin, H., And Kuroiwa, Y. 2004. Configuration processing of nexorades using genetic algorithms. Jour. of the Intl. Assoc. for Shell and Spatial Structures 45, 2, 99-108.

Brocato, M., And Mondardini, L. 2010. Geometric methods and computational mechanics for the design of stone domes based on Abeille's bond. In Advances in Architectural Geometry, Springer, 149-162.

CHILton, J. 1995. History of timber structures, Lecture E1. In STEP 2, Timber Engineering. STEP 2, Timber Engineering 2, E1-E13.

Chilton, J. 2009. Development of timber reciprocal frame structures in the UK. In Proceedings of IASS Symposium 2009: Evolution and trends in design, analysis and construction of shell and spatial structures, 1877-1884.

Cignoni, P., Pietroni, N., Malomo, L., And Scopigno, R. 2014. Fieldaligned mesh joinery. ACM Trans. on Graphics 33, 1 (January), art.11.

Douthe, C., And BAVErel, O. 2009. Design of nexorades or reciprocal frame systems with the dynamic relaxation method. Computers \& Structures 87, 21 (Nov.), 1296-1307.

Eigensatz, M., Kilian, M., Schiftner, A., Mitra, N., Pottmann, H., AND PAUly, M. 2010. Paneling architectural freeform surfaces. ACM Tran. on Graphics (SIGGRAPH) 29, 4, 45:1-45:10.

FU, C.-W., LAI, C.-F., HE, Y., AND COHEN-OR, D. 2010. K-set tileable surfaces. ACM Tran. on Graphics (SIGGRAPH) 29, 4, 44:1-44:6.

Gelez, S., AND SABy, V. 2011. Nexorades, facing an emergency situation. Intl. Jour. of Space Structures 26, 4 (Nov.), 359-362.

Grünbaum, B., And ShePhard, G. C. 1986. Tilings and patterns. W. H. Freeman \& Co.

Jiang, C., Wang, J., Wallner, J., And Pottmann, H. 2014. Freeform honeycomb structures. Computer Graphics Forum 33, 5. Proc. Symp. Geom. Processing.

Kohlhammer, T., And Kotnik, T. 2010. Systemic behaviour of plane reciprocal frame structures. Structural Engineering Intl. 21, 1, 80-86.

LARSEN, O. P. 2008. Reciprocal Frame Structures. Elsevier Science and Technology.

Li, X.-Y., Shen, C.-H., HuAng, S.-S., Ju, T., And Hu, S.-M. 2010. Popup: automatic paper architectures from 3D models. ACM Tran. on Graphics (SIGGRAPH) 29, 4, 111:1-111:9. 
Mitani, J., AND SuzuKi, H. 2004. Making papercraft toys from meshes using strip-based approximate unfolding. ACM Tran. on Graphics (SIGGRAPH) 23, 3, 259-263.

Panozzo, D., Block, P., And Sorkine-Hornung, O. 2013. Designing unreinforced masonry models. ACM Transactions on Graphics (proceedings of ACM SIGGRAPH) 32, 4, 91:1-91:12.

PARIgi, D., KirkeGaARd, P. H., And SAssone, M. 2012. Hybrid optimization in the design of reciprocal structures. In Proceedings of the IASS Symposium 2012: from spatial structures to spaces structures. 8 pages.

Pugnale, A., Parigi, D., Kirkegaard, P. H., And Sassone, M. 2011. The principle of structural reciprocity: history, properties and design issues. In IASS: Intl. Conference on Space Structures, 414-421.

SChWARTZBURG, Y., AND PAUly, M. 2013. Fabrication-aware design with intersecting planar pieces. Computer Graphics Forum (Proceedings of Eurographics 2013) $32,2$.

Sheffer, A., Lévy, B., Mogilnitsky, M., And Bogomyakov, A. 2005. ABF++: Fast and robust angle based flattening. ACM Tran. on Graphics 24, 2 (Apr.), 311-330.

Singh, M., AND SchAEFER, S. 2010. Triangle surfaces with discrete equivalence classes. ACM Tran. on Graphics (SIGGRAPH) 29, 46:1-46:7.

Song*, P., Fu*, C.-W., Goswami, P., Zheng, J., Mitra, N. J., And CohenOR, D. 2013. Reciprocal frame structures made easy. ACM Transactions on Graphics (SIGGRAPH) 29, 4. Article 94. (* joint first authors).

THÖNNISSEN, U., AND WERENFELS, N. 2011. Reciprocal frames - teaching experiences. Intl. Jour. Of Space Structures 26, 4, 369-372. (Rhino-script developed by Prof. Annette Spiro).

Umetani, N., IgARAshi, T., AND Mitra, N. 2012. Guided exploration of physically valid shapes for furniture design. ACM Tran. on Graphics (SIGGRAPH) $31,4,86: 1-86: 11$.

Whiting, E., OChSEndorf, J., And Durand, F. 2009. Procedural modeling of structurally-sound masonry buildings. ACM Tran. on Graphics (SIGGRAPH ASIA) 28, 5, 112:1-112:9.

Whiting, E., Shin, H., Wang, R., Ochsendorf, J., And Durand, F. 2012. Structural optimization of 3D masonry buildings. ACM Tran. on Graphics (SIGGRAPH ASIA) 31, 6, 159:1-159:11.

Xin, S.-Q., LaI, C.-F., Fu, C.-W., Wong, T.-T., He, Y., And Cohen-Or, D. 2011. Making burr puzzles from 3D models. ACM Tran. on Graphics (SIGGRAPH) 30, 4, 97:1-97:8. 
Yang, Y.-L., Yang, Y.-J., Pottmann, H., And Mitra, N. J. 2011. Shape space exploration of constrained meshes. ACM Tran. on Graphics (SIGGRAPH ASIA) 30, 6, 124:1-124:10. 